\title{
Population-Based Estimates of Chronic Conditions Affecting Risk for Complications from Coronavirus Disease, United States
}

\author{
Mary L. Adams, David L. Katz, Joseph Grandpre
}

We estimated that $45.4 \%$ of US adults are at increased risk for complications from coronavirus disease because of cardiovascular disease, diabetes, respiratory disease, hypertension, or cancer. Rates increased by age, from $19.8 \%$ for persons $18-29$ years of age to $80.7 \%$ for persons $\geq 80$ years of age, and varied by state, race/ ethnicity, health insurance status, and employment.

Data ata for China indicate that $81 \%$ of coronavirus disease (COVID-19) patients had mild cases, $14 \%$ had severe cases, and $5 \%$ had critical cases $(1,2)$. The overall case-fatality rate (CFR) in China was 3.8\% (3), but CFRs were higher for adults with chronic conditions of cardiovascular disease (CVD; CFR 13.2\%), diabetes $(9.2 \%)$, chronic respiratory disease $(8.0 \%)$, hypertension $(8.4 \%)$, and cancer $(7.6 \%)$, compared with $1.4 \%$ for patients with none of these conditions (3). Our objective for this study was to use populationbased US data to estimate the fraction of adults in the community who might be at increased risk for complications from COVID-19 because they reported any of the chronic conditions with a high CFR in China.

\section{The Study}

We used publicly available 2017 Behavioral Risk Factor Surveillance System (BRFSS) data (4) from telephone surveys of 444,649 randomly selected adults $\geq 18$ years of age in the 50 states and the District of Columbia (DC). Because the BRFSS includes only noninstitutionalized adults, residents of nursing homes and assisted living facilities are among those not surveyed. We chose to use 2017 data to include hypertension, which

Author affiliations: On Target Health Data LLC, Suffield, Connecticut, USA (M.L. Adams); True Health Initiative, Derby, Connecticut, USA (D.L. Katz); Wyoming Department of Health, Cheyenne, Wyoming, USA; (J. Grandpre)

DOI: https://doi.org/10.3201/eid2608.200679 was not addressed in 2018. Data were adjusted for the probability of selection and weighted to be representative of the adult population in each state by age, sex, race/ethnicity, marital status, education, home ownership, and telephone type. Weights and stratum variables needed for analysis were included.

We did not age-adjust results to reflect the age distribution of each state rather than a standard population. The median response rate for cell phone and landline surveys combined was $47.2 \%$, ranging from $33.9 \%$ in California to $61.1 \%$ in Utah (5). Reliability and validity of the BRFSS have been found to be moderate to high for many survey measures, in particular those used here, which can be checked versus medical records (6).

The key variable was a composite measure including adults reporting that they were ever told they had CVD (heart attack, angina, coronary heart disease, or stroke), diabetes, asthma, chronic obstructive pulmonary disease, hypertension, or cancer other than skin. We counted the number of chronic conditions for each respondent and adults who reported $\geq 1$ condition and were considered to be at heightened risk for complications from COVID-19. A secondary measure was receipt of a seasonal influenza vaccination in the past year as a rough estimate of potential demand for a COVID-19 vaccine when available.

Demographic measures included age group (18$29,30-39,40-49,50-59,60-69,70-79$, and $\geq 80$ years of age; we created these measures by combining 5 -year age groups provided in the dataset); self-reported race/ethnicity (non-Hispanic White, Black or African American, Hispanic of any race, American Indian/ Alaska native, Asian/Pacific Islander, and other); health insurance coverage (any kind of healthcare coverage, including health insurance, prepaid plans such as health maintenance organizations, or government plans such as Medicare, or Indian Health Service); employment status (employed or self-employed, out 
of work, homemaker, student, retired, or unable to work); and state of residence, which included DC.

We used Stata version 14.1 (StataCorp LP, https://www.stata.com) for analysis to account for the complex sample design of the BRFSS. We report point estimates and $95 \%$ CIs or population estimates by using weights, stratum, and primary sampling unit variables supplied in the dataset (4). Missing values were excluded from analysis.

Among 444,649 survey respondents, 48.7\% were male, $13.9 \%$ were $\geq 70$ years of age, $63.3 \%$ were white, $18.2 \%$ were retired, and $12.1 \%$ were uninsured. We obtained similar results for the study sample when 11,508 records that had missing values were removed. Overall, $45.4 \%$ (95\% CI $45.1 \%-45.7 \%$ ) of respondents fit the description of being at heightened risk for complications from COVID-19.

Among all adults, 26.7\% (95\% CI 26.5\%-27.0\%) reported 1 chronic condition, $12.0 \%$ (95\% CI $11.8 \%-$ $12.2 \%$ ) reported 2 chronic conditions, $4.7 \%$ (95\% CI $4.6 \%-4.8 \%$ ) reported 3 chronic conditions, and $2.0 \%$ $(95 \%$ CI $1.9 \%-2.1 \%)$ reported $\geq 4$ chronic conditions. Prevalence rates of separate chronic conditions were 8.5\% for CVD, $6.6 \%$ for chronic obstructive pulmonary disease, 9.1\% for asthma (active condition), 10.8\% for diabetes, $32.4 \%$ for hypertension, and $6.8 \%$ for cancer. Although the percentage of adults with any of the chronic conditions increased with age (Table1, https:/ / wwwnc.cdc.gov/EID/article/26/8/20-0679-T1.htm), more than half $(53.4 \%)$ of the total were $18-59$ years of age. Rates also varied by state, race/ethnicity, insurance status, and employment, but not by sex (Table 1).

State results obtained directly from Stata (Table 2) list the number of adults in each state at increased risk for complications and the percentage that number represents among all states. Results for reporting a seasonal influenza vaccination in the past year were $40.3 \%$ (95\% CI $40.0 \%-40.6 \%)$ for all adults, including $33.7 \%$ (95\% CI $33.3 \%-34.2 \%$ ) for adults who had none of the chronic conditions and $48.0 \%(95 \%$ CI $47.5 \%-48.5 \%$ ) for persons who had any of the 6 chronic conditions.

\section{Conclusions}

We estimated that $45.4 \%$ of US adults, with a wide range across age groups and states, might be at increased risks for complications from COVID-19 because of existing chronic conditions. The $18.7 \%$ who reported $\geq 2$ chronic conditions might be at even greater risk on the basis of other studies (7). Although complication rates increased with increasing age, $53 \%$ of those at greater risk for complications were $<60$ years of age. Preliminary data suggest that some of these same chronic conditions increase risk for COVID-19 complications in the United States (8). Another BRFSS study that omitted hypertension and used a different definition of risk estimated that $37.6 \%$ of US adults were at risk for complications (9).

Table 2. Number of adults with any of 6 chronic conditions
increasing risk for coronavirus disease complications and
percentage of total in each state, 2017 Behavioral Risk Factor
Surveillance System, United States
Surveillance System, United States*

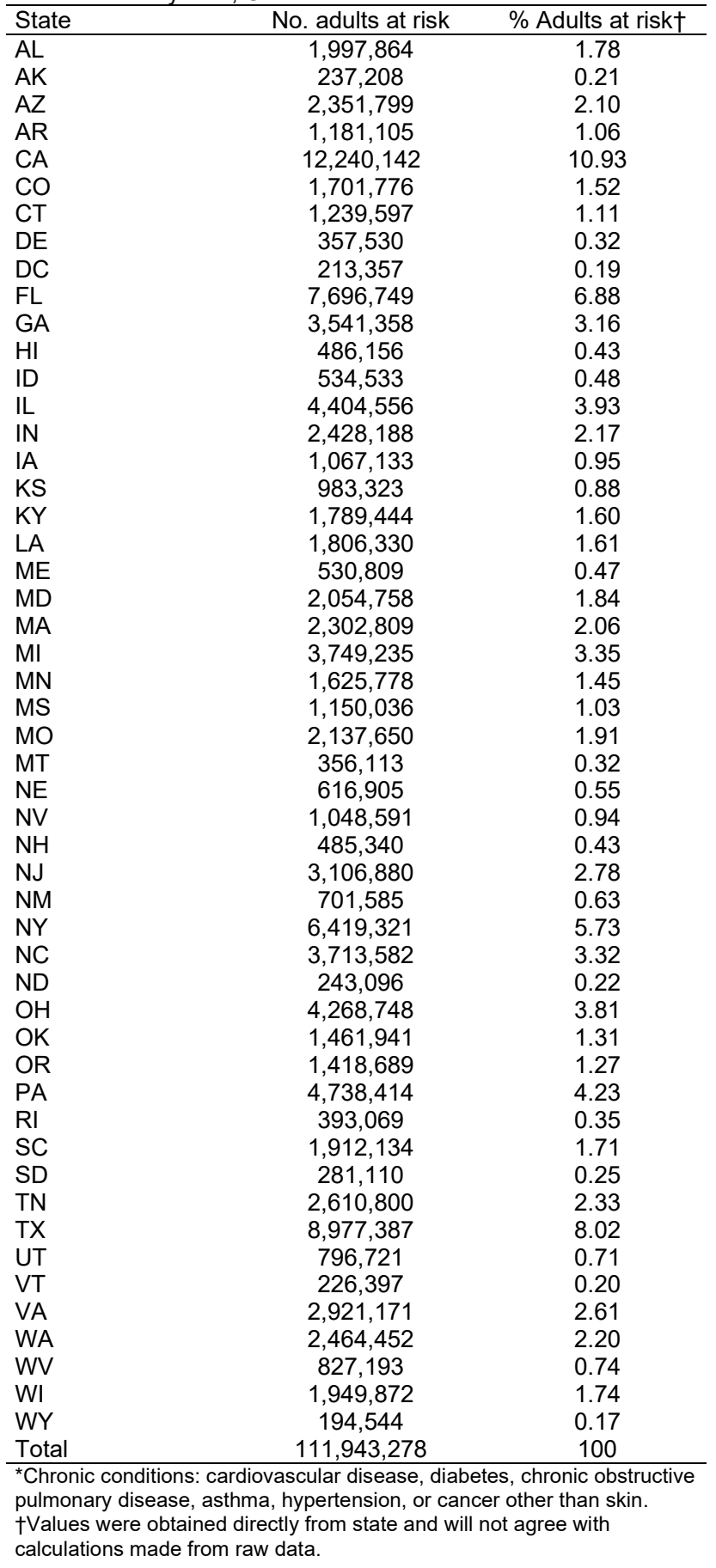


The list of chronic conditions used in our analysis is similar to groups at increased risk for seasonal influenza complications (10), except that the influenza group includes obese adults. Obesity is much lower in China than in the United States (11), which might account for that difference. Both lists include persons with chronic diseases for which behavioral risk factors have been well identified $(12,13)$. In particular, the 7 risk factors of smoking, sedentary lifestyle, obesity, diabetes, hypertension, high cholesterol, and inadequate fruit and vegetable consumption together contributed to an average of $41.4 \%$ of the burden of 5 of the 6 chronic conditions used in our study (all except cancer); obesity and smoking contributed the most to the burden (12). Results showing seasonal influenza vaccination rates $<50 \%$ are concerning. Although a vaccine specific for this coronavirus is currently unavailable, results for seasonal influenza vaccination suggest that it might not be widely used.

Our study does not address possible differences in contracting the disease, only the risk for development of complications among persons who have COVID-19 on the basis of results for China (1-3). Because we surveyed only noninstitutionalized adults, we excluded 1.3 million adults in nursing homes (14), which almost certainly underestimates risk. Data are self-reported, and reliability and validity can vary for different measures tested (6). However, as long as a respondent was told they had a chronic condition, validity was high. Age groups used for analysis did not match those used for weighting data, but that limitation should have a minimal effect on results. Low response rates could introduce bias but, as noted, validity appears high for the measures used in this study.

We estimated that $45.4 \%$ of US adults are potentially at increased risk for complications from COVID-19 because of chronic conditions that are, in turn, associated with common modifiable risk factors. Such estimates will vary depending on exact criteria used and the prevalence of the risk factors associated with the chronic conditions, along with age, state of residence, and other demographic factors.

Data collection, analysis, and interpretation for this study were supported by the Centers for Disease Control and Prevention (Grant/Cooperative Agreement no. 1U58DP006069-01).

\section{About the Author}

Ms. Adams is a consultant at On Target Health Data LLC, Suffield, CT. Her primary research interest is chronic diseases (including dementia) and their risk factors.

\section{References}

1. Wu Z, McGoogan JM. Characteristics of and important lessons from the coronavirus disease 2019 (COVID-19) outbreak in China: summary of a report of 72314 cases from the Chinese Center for Disease Control and Prevention. JAMA. 2020;323:1239 [Epub ahead of print]. https://doi.org/10.1001/jama.2020.2648

2. The epidemiological characteristics of an outbreak of 2019 novel coronavirus diseases (COVID-19) in China [in Chinese]. Zhonghua Liu Xing Bing Xue Za Zhi. 2020;41:145-51.

3. World Health Organization. Report of the WHO-China joint mission on coronavirus disease 2019 (COVID-19), February 28, 2020 [cited 2020 Apr 17]. https:/ / www.who.int/docs/ default-source/coronaviruse/who-china-joint-mission-oncovid-19-final-report.pdf

4. Behavioral Risk Factor Surveillance System (BRFSS) Survey Data and Documentation [cited 2020 Mar 3]. https:/ / www. cdc.gov/brfss/data_documentation/index.htm

5. Centers for Disease Control and Prevention. Behavioral Risk Factor Surveillance System 2017 Summary Data Quality Report, June 13, 2018 [cited 2018 Nov 16]. https:/ / www.cdc.gov/brfss/ annual_data/2015/pdf/2015-sdqr.pdfhttps://www.cdc.gov/ brfss/annual_data/2017/pdf/2017-sdqr-508.pdf

6. Pierannunzi C, Hu SS, Balluz L. A systematic review of publications assessing reliability and validity of the Behavioral Risk Factor Surveillance System (BRFSS), 2004-2011. BMC Med Res Methodol. 2013;13:49. https:/ / doi.org/10.1186/1471-2288-13-49

7. Sambamoorthi U, Tan X, Deb A. Multiple chronic conditions and healthcare costs among adults. Expert Rev Pharmacoecon Outcomes Res. 2015;15:823-32. https:/ / doi.org/10.1586/ 14737167.2015.1091730

8. Chow N, Fleming-Dutra K, Gierke R, Hall A, Hughes M, Pilishvili T, et al.; CDC COVID-19 Response Team. Preliminary estimates of the prevalence of selected underlying health conditions among patients with coronavirus disease 2019 United States, February 12-March 28, 2020. MMWR Morb Mortal Wkly Rep. 2020;69:382-6. https:/ / doi.org/10.15585/mmwr.mm6913e2

9. Koma W, Neuman T, Claxton G, Rae M, Kates J, Michaud J. How many adults are at risk of serious illness if infected with coronavirus? Kaiser Family Foundation, Washington, DC. March 2020 [cited 2020 Apr 17]. https://www.kff.org/ global-health-policy/issue-brief/how-many-adults-are-atrisk-of-serious-illness-if-infected-with-coronavirus/

10. Centers for Disease Control and Prevention. Influenza (flu): people at high risk for flu complications [cited 2020 Apr 17]. https://www.cdc.gov/flu/highrisk/index.htm

11. Wu Y. Overweight and obesity in China. BMJ. 2006;333:3623. https:// doi.org/10.1136/bmj.333.7564.362

12. Adams ML, Grandpre J, Katz DL, Shenson D. The impact of key modifiable risk factors on leading chronic conditions. Prev Med. 2019;120:113-8. https:/ / doi.org/10.1016/j.ypmed.2019.01.006

13. Adams ML, Grandpre J, Katz DL, Shenson D. Linear association between number of modifiable risk factors and multiple chronic conditions: Results from the Behavioral Risk Factor Surveillance System. Prev Med. 2017;105:169-75. https://doi.org/10.1016/j.ypmed.2017.09.013

14. Centers for Disease Control and Prevention. National Center for Health Statistics. Nursing home care [cited 2020 Apr 17]. https://www.cdc.gov/nchs/fastats/nursing-home-care.htm

Address for correspondence: Mary L. Adams, On Target Health Data LLC, 247 N Stone St, West Suffield, CT 06093, USA; email: madams.ontargethealthdata@gmail.com 